\title{
Wolf-Rayet stars in Active Galactic Nuclei
}

\author{
Robin J.R. Williams and John E. Dyson \\ Department of Physics and Astronomy, University of Leeds, \\ Leeds LS2 9JT, UK \\ Judith J. Perry \\ Institute of Astronomy, University of Cambridge, \\ Cambridge CBЗ OHA, UK
}

Starbursts, black holes and AGN have strong observational links, as discussed elsewhere in these proceedings. Perry \& Dyson (1985 (PD), see also Perry 1994) studied the role of shocks around supernovae and stellar wind bubbles in the nuclei of active galaxies. Both the ejecta and the ambient ISM are initially shocked to high temperatures. PD found that while the shocked gas is maintained at high pressure by ram pressure, it cools rapidly, to then produce the observed optical and UV emission lines. The mass supply rate from the nuclear starburst, inferred from the strength of the emission lines, tallies well with that required by an accreting black hole to generate the observed luminosity. A symbiosis between a starburst stellar cluster and an accreting black hole naturally generates the observational features associated with QSOs.

Wolf-Rayet stars are an important constituent of such metal-rich starburst clusters. In clusters with metal abundances typical of galactic nuclei, the WR phase is responsible for a substantial fraction of the mass loss from massive stars. The high metallicity of the gas in WR winds promotes Bremsstrahlung and line emission, so shocked WR ejecta will be seen preferentially in such nuclei. In particular, Seyfert nuclei are sufficiently small that supernovae will be infrequent, and those that do occur will run rapidly through them. Therefore stellar wind sources of shocked gas will dominate. WR stars may also have important consequences for the abundances of line-emitting gas in all AGN, particularly in producing high metal abundances in cool gas at the early cosmic times to which AGN are now observed. The strong neon lines seen in the UV (Hamann et al. 1998) are also suggestive of the importance of mass input by WR stars.

Compared to well-studied WR/OB binaries, WR bow-shocks in AGN will be rather larger, and the WR ejecta flowing through them will be on the margins of cooling. The external radiation field in AGN serves both to enhance the cooling of the shocked gas somewhat, and also to irradiate any gas which cools in the bow-shock and hence to enhance its line emission.

Stevens et al. (1992) found that strongly cooling shocks were highly unstable, but classified shocks with $t_{\text {cool }} / t_{\text {dyn }}=\chi \geq 1$ as effectively adiabatic. In the Figure, a simulation of a WR bow-shock with a marginally-cooling WR wind is shown. Gas which shocks at the apex maintains its small angular momentum 


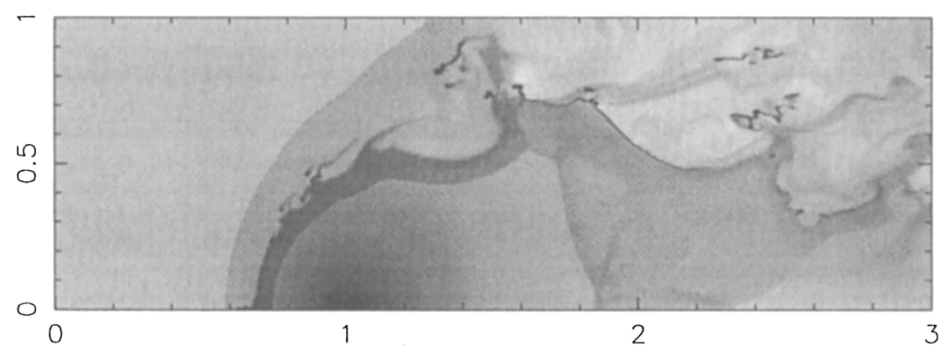

Figure 1. A simulation with a WR wind at $10^{3} \mathrm{~km} \mathrm{~s}^{-1}$ for which $\chi=2.25$ (the stellar mass loss rate assumed is $10^{-5} \mathrm{M}_{\odot} \mathrm{yr}^{-1}$ and the standoff distance is $2.25 \times 10^{14} \mathrm{~cm}$, so the density in the cool gas at stagnation is $n_{\mathrm{H}}=3 \times 10^{10} \mathrm{~cm}^{-3}$, comparable to that found from models of the BELR). The impinging wind has a velocity $4 \times 10^{3} \mathrm{~km} \mathrm{~s}^{-1}$, and shocks to a temperature to high for it to cool. This simulation was run using an adaptive grid code (AQUALUNG, Williams 1999), with an effective $512 \times 1536$ grid.

as it advects around the shock, and hence takes a considerable period to escape. The net effect is that gas which joins the shock close to the apex can cool even when $\chi=2.25$. Resolving the shocked layer suppresses the Kelvin-Helmholtz instability, as it allows the simulated flow to capture the small transverse velocity of the gas close to the contact discontinuity.

The cooled gas has a high-density contrast with the surrounding hot shocked gas, and tends to collapse into small clumps and then escape more-or-less ballistically. These cool, dense clumps are then ablated at an increasing rate as their velocity with respect to the local hot gas increases. The form of the flows suggests that in three dimensions, the covering area of the fragmentary clumps may be significantly lower than the geometrical area of the bow-shocks.

In summary, even with marginal cooling, we find that WR bow-shocks may be important sources of the $10^{4} \mathrm{~K}$ gas which produces the optical/UV emission lines in AGN.

For full details, see Perry et al. (1999).

\section{References}

Hamann, F., Cohen, R.D., Shields, J.C., Burbidge, E.M., Junkkarinen, V., Crenshaw, D.M. 1998, ApJ 496, 761

Perry, J.J., Dyson, J.E. 1985, MNRAS235, 665 (PD)

Perry, J.J. 1994, in: G.V. Bicknell, M.A. Dopita \& P.J. Quinn (eds), The Physics of Active Galaxies, ASP-CS 54, 417

Perry, J.J., Williams, R.J.R., Dyson, J.E. 1999, in preparation

Stevens, I.R., Blondin, J.M., Pollock, A.M.T. 1992, ApJ 386, 265

Williams, R.J.R. 1999, in preparation 